\title{
Extranodal NK/T-Cell Lymphoma, Nasal Type Overlapping Aggressive NK-Cell Leukemia in Course of Progression
}

\author{
Ivan PETKOVIĆ ${ }^{1,2}$, Slavica STOJNEV ${ }^{1,3}$, Aleksandar POPOVIĆ2, Miljan KRSTIĆ ${ }^{1,3}$, \\ Ivica PEJČIÍ́ ${ }^{1,2}$ \\ ${ }^{1}$ University of Niš, Faculty of Medicine, Department of Oncology \\ ${ }^{2}$ Clinical Centre Niš, Oncology Clinic \\ ${ }^{3}$ Clinical Center Niš, Centre for Pathology, Niš, SERBIA
}

To the Editor,

We present a case of extranodal NK/T-cell lymphoma (ENKL) with leukemic transformation overlapping aggressive NK-cell leukemia (ANKL) in the course of progression.

ENKL is an aggressive neoplasm with putative NK-cell, and rarely cytotoxic T-cell origin. In the majority of cases, it presents as locally destructive mid-facial necrotizing lesions, particularly involving nasal/ paranasal region. ${ }^{1,2}$ Extranasal involvement (eyes, gut, lungs, gonads, skin, or other) have been reported characterized by a highly aggressive course, chemo-refractoriness, resulting in short survival. ${ }^{3}$ The uniform angiocentric, and angiodestructive growth pattern with prominent necrosis are major histological hallmarks. Almost 100\% of malignant NK-cells are Epstein-Baar virus (EBV) positive. ${ }^{1}$ The membrane CD3 negativity and cytoplasmic $\mathrm{CD} 3 \varepsilon$ chain positivity with the expression of NK-cell markers are typical. The bone marrow involvement is uncommon at presentation. ${ }^{1}$ Clinical stage I/II vs. III/IV are clearly delineated in terms of survival. ${ }^{4}$ Multivariate analysis pointed non-nasal type, poor performance status (PS), advanced stage, and numbers of extranodal involvement as a significant for poor outcome. ${ }^{5}$ The leu- kemic transformation of ENKL to ANKL is an uncommon biological course of the disease. ${ }^{6}$

A 58-year-old male presented with rapidly growing ulcerative mass of a hard and soft palate with the extension to the nasal cavity, causing nasal congestion, local severe pain, and difficulty in swallowing. He reported no B symptoms. No mosquito bites hypersensitive reactions were reported by patient. There was no data about chronic active EBV infections from previous medical records. The maxillofacial surgeon performed biopsy of the local tumor. Histopathology revealed ENKL, nasal type with immunohistochemical profile: cytoplasmic CD3e+, CD56+, CD2+, EBV+, Ki67 > $80 \%$, while pan B markers were negative (Figure $1 \mathrm{~A}, \mathrm{~B}, \mathrm{C}, \mathrm{D})$. Subsequent to the pathology report the patient underwent staging procedures at our institution. The head/neck MSCT scans showed a gingival infiltrative mass on the medial right side of the maxilla $26 \times 14 \mathrm{~mm}$ in diameter. Gingival tumor medially merged with massive infiltration of the soft palate up to $37 \times 36 \mathrm{~mm}$ in diameter. There was also a tumor mass of the oropharynx $29 \mathrm{~mm}$ in size. MSCT scans of the thorax, abdomen and pelvis were remarkable. 


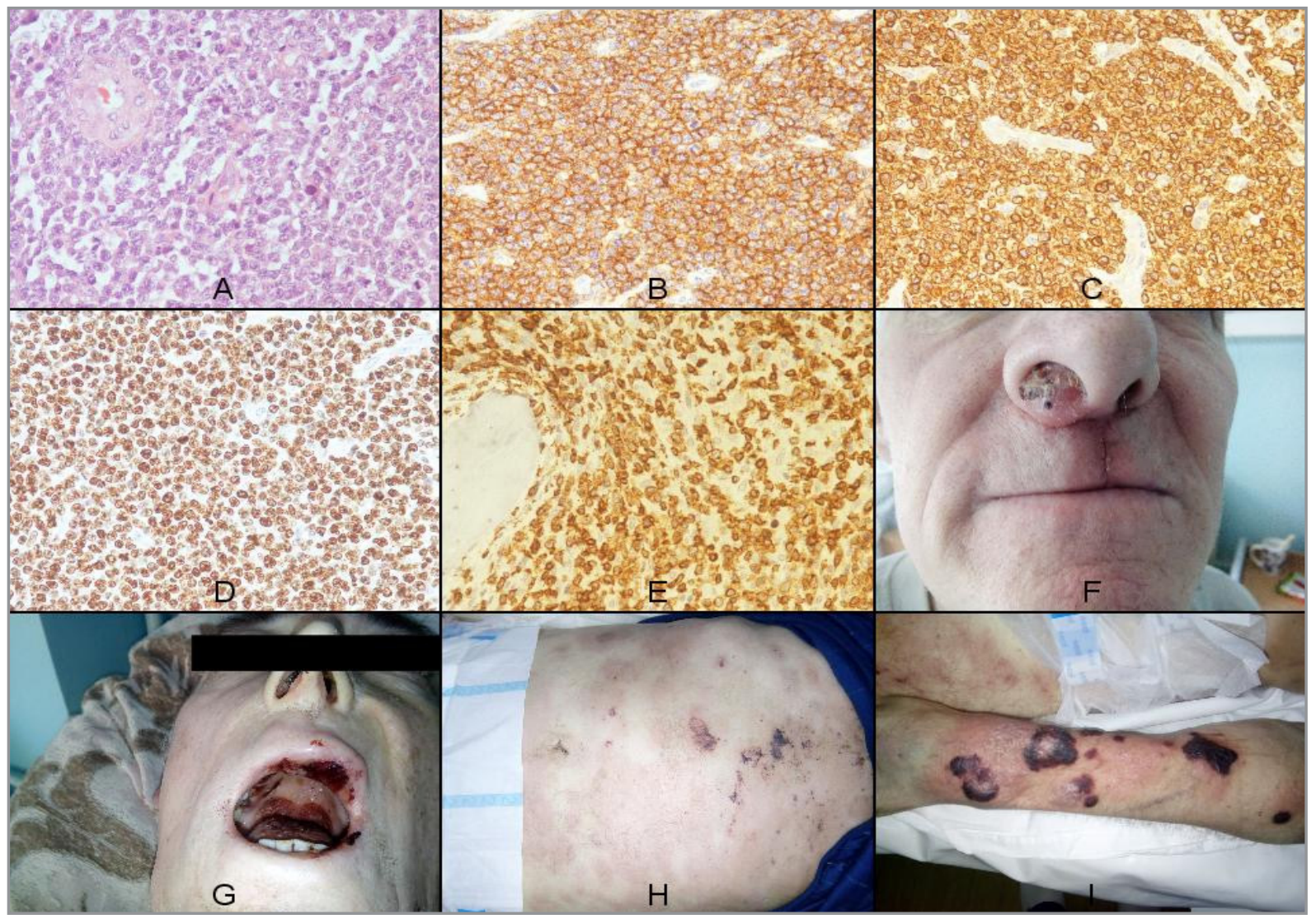

Figure 1. Extranodal NK/T cell lymphoma, nasal type-micromorphological and clinical presentation. (A) Atypical medium sized lymphoid infiltrate with distinctive angiocentric and angioinvasive growth. Neoplastic cells display vesicular nuclei with irregular borders and inconspicuous nucleoli, pale cytoplasm frequent mitotic figures, and are admixed with numerous apoptotic bodies (HE, x40); (B) Diffuse immunohistochemical expression of CD56, and (C) CD3E; (D) Ki67 nuclear labeling in the majority of tumor cells, indicating very high proliferative activity; (E) Massive bone marrow infiltration by CD3E+ atypical lymphoid cells marking the transformation into aggressive NK/T cell leukaemia; (F) Ulcerative tumor mass obstructing the nasal cavity; (G) Destructive lesions in oral cavity in leukaemic phase of the disease; (H, I) Extensive neoplastic infiltrates of the skin accompanied by hematomas and suffusions.

Hematological and biochemical parameters were inconspicuous, except elevated $\mathrm{LDH} 568 \mathrm{U} / \mathrm{L}$, CRP $30 \mathrm{mg} / \mathrm{L}$, and $\beta 2$-microglobulin $3.20 \mathrm{mg} / \mathrm{L}$. The virological serology determined HIV-, HCV-, HBsAg-, EBV IgG+ status. Bilateral bone marrow biopsy revealed regular hematopoiesis with no infiltration of ENKL (CD3E-, CD56-, EBV-). The patient was assigned II A clinical stage. He received 3 induction courses of Ifosfamide, Methotrexate, Etoposide + high dose Dexamethasone (VIM/ Dexa) regimen. Although the incorporation of LAsparaginase (L-Aspa) represents the backbone of current management ${ }^{7}$, it has not been formally approved yet in Serbia. Severe hematologic and infectious adverse events accompanied the induction setting. Even though the patient barely tolerated therapy, he still received 3 cycles obtaining partial remission (PR). The further management plan was to conduct the concomitant chemo-radiotherapy 60-70 Gy with 3 x Cisplatin $100 \mathrm{mg} /$ $\mathrm{m}^{2}$. However, the execution was incomplete due to the patient's renal impairment, and low adherence, thus he actually received $3 \times$ Cisplatine $40 \mathrm{mg} / \mathrm{m}^{2}$ +36 Gy of radiotherapy. Almost immediately after completing of upfront management, the disease progressed. In a course of progression, the patient developed refractory pancytopenia, escalation of B symptoms, local nasal tumor progression, multiple skin infiltrations with diffuse hemorrhages. Peripheral blood smear detected immature large lymphoid cells resembling NK-cells. Level of LDH was over $8000 \mathrm{U} / \mathrm{L}$. We performed a trephine biopsy under platelet transfusion. The pathology report depicted a massive bone marrow leukemic infiltration (> 
$80 \%$ ) with a picture of a "packed marrow". No haemophagocytic cells were detected. The immunophenotype of blasts was: CD3ع+, CD56+, and CD16- (Figure 1 E, F, G, H, I). The patient's PS deteriorated promptly accompanied by permanently present exhausting B symptoms. He was palliated with Dexamethasone, Cyclophosphamide, and Vincristine with unsatisfactory results, and died from sepsis and multiorgan failure.

Bone marrow involvement in early stage ENKL is uncommon, detected in $\approx 7 \%$ of cases. ${ }^{1}$ However, Lee et al. conducted a trial in 91 patients with localized stage I/II ENKL by using EBV-encoded RNA in situ hybridization (EBER ISH) techniques for trephine biopsy specimens. All patients had bilateral trephine biopsies so they examined a total of 182 specimens. The results obtained showed that $18.7 \%$ of patients had EBER ISH positive bone marrow specimen indicating low burden infiltration. ${ }^{8}$ These results provide a powerful tool for more accurate staging in early ENKL. Inspite the progressiveness of the results, they have never been implemented in a routine practice according to all international guidelines for lymphoma staging (ESMO, NCCN). Immunohistochemical analysis $(\mathrm{CD} 3 \varepsilon, \mathrm{CD} 56, \mathrm{EBV})$ is regarded sufficient and remains a mainstay in assessment of bone marrow involvement by ENKL. Our patient had regular bone marrow with negative stains at presentation. However, in a course of progression, he developed pancytopenia accompanied by overpopulated bone marrow with typical $\mathrm{CD} 3 \varepsilon+$, CD56+, CD16- blasts, supporting our assessment of leukemic transformation into ANKL. The data considering the development of ANKL under the initial diagnosis of early stage ENKL in a course of progression are scarce. Whether ANKL is a distinct neoplasm or a leukemic variant of ENKL remains doubtful. ${ }^{9,10}$ Given its extreme rarity and a lack of information, it seems that ANKL and systemic ENKL are indistinguishable conditions. If we look at the immunophenotype data of ANKL (de novo or acquired) no difference can be distinguished from ENKL except perhaps that ANKL frequently expresses CD16 in $\approx 75 \%$ cases. ${ }^{1}$ Nevertheless, the remaining $25 \%$ of cases are CD16 negative which does not exclude the diagnosis of ANKL. Skin involvement is uncommon in de novo ANKL, but the skin may be a site of infiltration like every other organ. ${ }^{1}$ Our case demonstrates that skin may be a site of involvement. ENKL is rare in people outside Asia, and the development of ANKL in the field of previous ENKL is extremely rare, giving this case a unique significance. The natural flow of leukemic disease completely resembled ANKL: fulminant clinical course complicated with coagulopathy and multiorgan failure. The overall survival was less than 2 months.

A number of questions remain open as to whether it was a primary refractory disease, or whether the progression with leukemic transformation was at least partially dependent on treatment? Was the ANKL transformation a natural course of this specific case as an independent event regardless of the treatment modality and to what extent did the omission of L-Aspa influenced the outcome? Regardless of the treatment modality, no defined predictive biomarkers have been developed for the leukemic evolution of the primary ENKL, nasal type. Therefore, the case we presented to the scientific community depicts one extremely rare course of a disease especially in non Asian geographical area, which is unique.

\section{REFERENCES}

1. Swerdlow SH, Campo E, Pileri SA, et al. The 2016 revision of the World Health Oranization classification of lymphoid neoplasms. Blood 127: 2375-2390, 2016.

2. Suzuki R. Leukemia and lymphoma of natural killer cells: Review. J Clin Exp Hematop 4: 51-70, 2005.

3. Park S, Ko YH. Epstein-Barr virus-associated T/ natural killer-cell lymphoproliferative disorders. J Dermatol 41: 29-39, 2014.

4. Petković I, Popović A, Džunić M, Pejčić I. Nodal and extranodal peripheral T/NK-cell neoplasms: Current aspects. Acta Fac Med Naiss 37: 99-120, 2020.

5. Suzuki R, Suzumiya J, Yamaguchi M, et al. Prognostic factors for mature natural killer (NK) cell neoplasms: aggressive NK cell leukemia and extranodal NK cell lymphoma, nasal type. Ann Oncol 21: 1032-1040, 2010.

6. Ishida F. Aggressive NK-Cell Leukemia. Front Pediatr 10: 292, 2018.

7. d'Amore F, Gaulard P, Trümper L, et al. Peripheral T-cell lymphomas: ESMO clinical practice guidelines for diagnosis, treatment and follow-up. Ann Oncol 26 suppl 26: 108-115, 2015. 
International Journal of Hematology and Oncology

8. Lee J, Suh C, Huh J, et al. Effect of positive bone marrow EBV in situ hybridization in staging and survival of localized extranodal natural killer/T-cell lymphoma, nasal-type. Clin Cancer Res 13: 3250-3254, 2007.

9. Quintanilla-Martinez L, Jaffe ES. Commentary: aggressive NK cell lymphomas: insight into the spectrum of NK cell derived malignancies. Histopathology 37: 372-374, 2000.

10. Xiaoke J, Xu Y, Zhang J, et al. Aggressive natural killer cell leukemia or extranodal NKTT cell lymphoma? A case with nasal involvement. Diagn Pathol 12: 45, 2017.

\section{Correspondence:}

Ivan PETKOVIĆ, M.D, Ph.D.

University of Niš, Faculty of Medicine

Department of Oncology, Niš, Serbia

Bulevar Dr Zorana Đinđića 48

18000 Niš, SERBIA

Tel: +381063 8020581

e-mail: ivan.petkovic@medfak.ni.ac.rs

\section{ORCIDs:}

Ivan Petković

0000-0002-9110-4117

Slavica Stojnev

0000-0001-5058-9720

Aleksandar Popović

0000-0003-2279-4721

Miljan Krstić

0000-0003-2634-3450

Ivica Pejčić

0000-0001-9107-1557 